\title{
PROPORCIONALIDADE E SEMELHANÇA: APRENDIZAGEM VIA OBJETOS DE APRENDIZAGEM
}

Alcibiades Gazzoni - Centro Universitário Franciscano - agazzoni@unifra.br

Ana Paula Canal - Centro Universitário Franciscano - anapala@unifra.br

Gilse A.Morgental Falkembach - Centro Universitário Franciscano gilsemf@terra.com.br

Leandra Anversa Fioreze - Centro Universitário Franciscano - leandra@ unifra.br

Leila Brondani Pincolini - Centro Universitário Franciscano - leila@ unifra.br

${ }^{1}$ Rodrigo Antoniazzi - Centro Universitário Franciscano rodrigoantoniazzi@yahoo.com.br

\section{Resumo}

Este artigo aborda a concepção e desenvolvimento do Objeto de Aprendizagem: Proporcionalidade e Semelhança.

Palavras Chaves: Objetos de Aprendizagem - Objetos Educacionais - Proporcionalidade

\section{Proportionality and Likeness: learning through learning objects}

\section{Abstract}

This article approaches the conception and development of the Learning Object: Proportionality and Likeness.

Keywords: Learning Objects - Educational Objects - Proportionality

\section{Introdução}

Com a evolução e o avanço das Novas Tecnologias da Informação e Comunicação (NTICs) o computador passou a ser um meio de ensino e tem acelerado o desenvolvimento do aprendizado eletrônico (e-learning) possibilitando a utilização de conteúdos didáticos estruturados e mais organizados. Esses conteúdos podem ser disponibilizados na rede em diferentes formatos e podem subsidiar as modalidades de ensino presencial, semi-presencial e a distância.

$\mathrm{O}$ ensino presencial é o ensino tradicional que apoiado nas tecnologias digitais permite ensinar as disciplinas de conteúdos áridos, que exigem abstrações, por meio de simulações, de imagens, sons e vídeos para aumentar a assimilação e a fixação desses conteúdos. Semi-presencial é o ensino que compreende aulas presenciais e à distância com o auxílio da telemática. A Educação a Distância, mediada pelas tecnologias digitais, é o ensino via um Ambiente Virtual de Aprendizagem que com os meios de comunicação disponíveis permite ensinar e aprender não mais limitados a uma sala de aula. Portanto, qualquer modalidade de ensino pode usufruir dos recursos oferecidos pelas novas tecnologias entre eles a Internet.

Os recursos oferecidos pelas tecnologias digitais possibilitam criar materiais didáticos que podem estimular o aprendiz tornando-o um cúmplice do processo de aprendizagem, engajando-o no processo do seu desenvolvimento e o professor se torna um facilitador desse processo. Os Objetos de Aprendizagem (Learning Objects, ou Educational Objects) surgem como um importante elemento para auxiliar no desenvolvimento de cursos subsidiados pelos recursos eletrônicos.

Este trabalho trata da concepção e desenvolvimento do Objeto de Aprendizagem Proporcionalidade e Semelhança por uma equipe interdisciplinar do Centro Universitário Franciscano - Santa Maria para o RIVED, Rede Internacional Virtual de 
Educação. Esse objeto foi premiado no concurso promovido pelo MEC através da Secretaria de Educação a Distância que envolveu alunos da graduação.

\section{Objetos de Aprendizagem}

A expressão Objetos de Aprendizagem significa material didático digital, com a característica da padronização no armazenamento e reutilização, ou seja, seu uso pode ser compartilhado o que exige que ele tenha uma estrutura modular associada com o desenvolvimento direcionado para a web. Pode ser formado por uma lição, pelo conteúdo de uma aula, de um curso, um programa de treinamento, uma unidade curricular ou uma atividade didática qualquer. É sempre formado por um conteúdo a ser aprendido. Portanto, deve levar em conta todos os procedimentos pedagógicos que vão desde a escolha do conteúdo a ser apresentado e das estratégias mais adequadas para fazê-lo, até a compreensão do processo de ensino e aprendizagem e das interações entre o aluno envolvido nesse processo e o conteúdo, através de um meio informatizado.

Objetos de aprendizagem, objetos educacionais, objeto independente, objeto de comunicação são sinônimos e se referem a um material didático digital, utilizado com propósitos educacionais, ou seja, é um courseware que deve obedecer a uma padronização para ser reutilizável. Segundo Wiley, 2000,

"objetos de aprendizagem são elementos de um novo tipo de instrução baseada em computador construído sobre um novo paradigma da Ciência da Computação. Eles permitem aos designers instrucionais a construção de pequenos componentes instrucionais os quais podem ser reutilizados inúmeras vezes em diferentes contextos de aprendizagem. Eles são geralmente entendidos como entidades digitais derivados da internet, e que podem ser acessados e utilizados por qualquer número de pessoas simultaneamente."

ou seja, Objeto de Aprendizagem é definido como uma entidade digital que pode ser usada, re-usada ou referenciada durante o ensino com suporte tecnológico. O organismo de padronização IEEE - Instituto de Engenheiros Elétricos e Eletrônicos - responsável pela definição de diversos padrões utilizados pela indústria eletro-eletrônica, por meio de seu Comitê de Padrões de Tecnologia de Aprendizagem em 2000 definiu objetos de aprendizagem como: "qualquer entidade, digital ou não, que pode ser utilizada, reutilizada ou referenciada durante o processo de aprendizagem que utilize tecnologia". Esta definição, muito usada na literatura sobre Objetos de Aprendizagem, é de pouco uso prático e, não limita o desenvolvimento do mercado de e-learning. Sua generalização permite que qualquer material seja considerado um Objeto de Aprendizagem, bastando que este seja utilizado em algum processo de ensino com base tecnológica. Segundo Handa e Silva, 2003, o Instructional Management System (IMS), consórcio de especificações dos fabricantes de softwares educacionais, consideram os Objetos de Aprendizagem com base na teoria de orientação a objetos, que é utilizada no desenvolvimento de sistemas computacionais, como sendo "um conjunto de rotinas $e$ estruturas de dados que interagem com outros objetos." Nos Objetos de Aprendizagem, o "objeto" serve para encapsular ou "armazenar" materiais digitais, transformando-os em módulos reutilizáveis de fácil manipulação segundo Downs, 2001.

A base teórica da orientação a objetos para subsidiar a criação de Objetos de Aprendizagem permite uma organização no desenvolvimento de materiais didáticos digitais facilitando a definição de padrões para a interoperabilidade entre diversos Objetos de Aprendizagem desenvolvidos independentemente. No entanto, ainda suscita críticas sobre as diversas definições existentes e Sosteric e Hesemeier, 2001, em um artigo concluíram que: "objetos de aprendizagem podem ser um arquivo digital com 
imagens, gráficos, vídeos, sons ou qualquer ferramenta usada como recurso educacional e que contenha sugestões sobre o contexto de sua utilização". Mesmo que essa definição seja incompleta serve como definição real de Objetos de Aprendizagem. Segundo Handa e Silva, 2003, essa definição não é tão genérica como a da IEEE e nem tão técnica quanto a teoria de orientação a objetos, mas engloba-as, não limitando o desenvolvimento de Objetos de Aprendizagem ao seu escopo e afirmando a necessidade de considerar os aspectos educacionais. Na verdade, entende-se por Objetos de Aprendizagem qualquer material ou recurso digital com fins educacionais.

As 2 organizações IEEE e IMS consideram as seguintes características como comuns aos objetos de aprendizagem: re-usabilidade, portabilidade, modularidade, metadados e interatividade.

- Re-usabilidade: essa característica faz com que os Objetos de Aprendizagem, como módulos básicos, sejam utilizados de diferentes formas, para trabalhar conteúdos diferentes em contextos diferenciados, ou seja, um Objeto de Aprendizagem deve permitir o seu uso em diversos cursos;

- Portabilidade: é a capacidade que um objeto tem de ser executado em diferentes plataformas de trabalho (sistemas operacionais), ou seja, um Objeto de Aprendizagem deve poder ser "transportado" para diversas plataformas;

- Modularidade: se refere à forma dos Objetos de Aprendizagem que deve ser em módulos independentes e não seqüenciais para poderem ser usados em conjunto com outros recursos e em diferentes contextos. Um Objeto de Aprendizagem é parte de um curso completo, podendo conter outros objetos de aprendizagem ou estar contido em um ou mais objetos ou em um ou mais cursos.

- Metadados: significa dados sobre dados. É a descrição completa dos atributos do objeto para a catalogação, obedecendo a padrões para a indexação, pesquisa e recuperação dos objetos, tornando-o compreensível para as diversas plataformas. As informações são: título, autor, data, publicação, palavras chaves, descrição, objetivos, características que mostra como, quando e por quem o objeto foi desenvolvido, armazenado e como está formatado. Os padrões de metadados mais comuns são: Learning Object Metadata (LOM) da IEEE e o Sharable Content Object Reference Model (SCORM)

- Interatividade: é uma das características mais importantes, pois se refere à interação do aluno com o objeto. A interação pode ser ativa ou não segundo a concepção do objeto.

Além das características acima os Objetos de Aprendizagem devem possuir ainda os seguintes atributos:

- Flexibilidade: eles são construídos em módulos com início, meio e fim, portanto, são flexíveis, podendo ser reutilizados sem manutenção, essa capacidade apresenta a vantagem de utilizar conhecimentos já escritos e consolidados;

- Facilidade para atualização: como todos os dados, relativos ao objeto, estão em um mesmo Banco de Dados a atualização feita através de correções e aperfeiçoamentos é simples;

- Customização: como os objetos são independentes, podem ser usados em cursos distintos (graduação, especialização ou qualquer outro tipo decurso) arranjados da maneira que mais convier, equivale à portabilidade; 
- Interoperabilidade: o armazenamento de Objetos de Aprendizagem é padrão. Isso cria mais uma vantagem que é a interoperabilidade, ou seja, a reutilização dos objetos não apenas em nível de plataforma de ensino, mas em nível mundial;

- Aumento da qualidade do ensino: o objeto sendo reutilizado diversas vezes em diversos cursos e sendo melhorado, a sua consolidação cresce e melhora significativamente a qualidade do ensino;

- Indexação e procura: a padronização dos objetos visa também facilitar a procura por um determinado objeto em qualquer banco de objetos que esteja disponível.

Todas essas peculiaridades mostram que o modelo Objetos de Aprendizagem vem para facilitar e melhorar a qualidade do ensino, proporcionando aos tutores, alunos e professores diversas ferramentas facilitadoras. Já existem diversos Banco de Dados (Banco de Objetos) principalmente americanos, nas mais diversas áreas de ensino utilizando as mais variadas mídias como imagens, animações, vídeos e applets para ensinar. É importante salientar que não apenas estes tipos de mídias podem ser utilizados, mas qualquer mídia que possa se veicular através da Internet, como por exemplo, documentos VRML (Realidade Virtual), arquivos documentos de todos os tipos (doc e txt), arquivos do tipo hipertexto (html) entre outros.

Segundo Tarouco, 2003, para se entender melhor o que são Objetos de Aprendizagem é possível fazer uma analogia com peças LEGO, com as quais se podem construir outros objetos que por sua vez também podem ser usados como peças de uma montagem maior e assim sucessivamente. Os Objetos de Aprendizagem podem ser usados para apoiar o processo de qualquer modalidade de ensino. O importante é saber onde encontrar os objetos adequados à proposta pedagógica usada pelo professor. Hoje existem repositórios de Objetos de Aprendizagem de diversas áreas tanto no Brasil como no exterior, tal como MERLOT, LABVIRT, RIVED e CESTA.

No Brasil existe um grupo de pesquisadores trabalhando nessa área. Na UFRGS, no CINTED (Centro Interdisciplinar de Novas Tecnologias na Educação) existe o CESTA (Coletânea de Entidades de Suporte ao uso de Tecnologia na Aprendizagem) que foi idealizado para sistematizar e organizar o registro dos objetos educacionais que vem sendo desenvolvidos pela equipe de Pós-Graduação em Informática na Educação. Considerando-se as mais variadas opções para a EAD isto é especialmente relevante, pois o ambiente de aprendizagem pode interagir diretamente com o repositório, selecionando e recuperando objetos, combinando-os de modo a compor unidades de suporte à aprendizagem que atendam as necessidades individuais de cada estudante. $\mathrm{O}$ Mec subsidia o projeto RIVED, Rede Internacional Virtual de Educação que oferece cursos para capacitar equipes interessadas, das universidades federais, na produção de Objetos de Aprendizagem, segundo as normas do projeto. $\mathrm{O}$ curso discute o impacto da tecnologia na Educação, planeja material didático reutilizável para uso em cursos de EAD.

A utilização de Objetos de Aprendizagem vem crescendo significativamente à medida que melhora o modo como os objetos são armazenados e distribuídos na Internet. A tendência é que os Objetos de Aprendizagem tornem-se um padrão mundial de troca de informações entre sistemas de ensino a distância e sendo unidades digitais disponibilizadas na Internet, podem ser criadas cooperativamente/colaborativamente e acessados de forma simultânea, a partir de um repositório.

\section{Concepção e desenvolvimento do Objeto de aprendizagem}

Segundo Falkembach, 2005, o processo de concepção e desenvolvimento de um Objeto de Aprendizagem, ou seja, de um material didático digital engloba o planejamento, a modelagem, a implementação e a distribuição. O planejamento envolve a escolha do 
tema a ser trabalhado e deve responder algumas questões como: qual o objetivo do Objeto de Aprendizagem? Qual o público alvo? Como o conteúdo será apresentado? Quando e como o objeto será usado? Quais os resultados esperados? Como o aluno vai interagir com o objeto? O assunto foi escolhido a partir de Tinoco, 1996 que afirma que é importante para um aluno perceber que tudo o que precisa ser construído antes é feito um modelo. Na vida real, para se construir uma casa, para um estilista criar sua coleção, para uma indústria produzir um aparelho eletrodoméstico ou de qualquer outra área, é preciso ser projetado. Os profissionais que fazem isso são técnicos, engenheiros, projetistas, desenhistas e outros. Ao se construir uma casa, é necessário, antes projetar a obra. As costureiras fazem uma roupa a partir do modelo feito pelo estilista. Ao se comprar um apartamento antes se olha o projeto do edifício onde se localiza o apartamento. Isso reporta para a importância de entender a relação entre o modelo e o objeto real. Daí a escolha do tema. Por sugestão dos professores da Matemática optouse por trabalhar essas noções a partir da ampliação e/ou redução de fotos. Escolhido o assunto e com base nas respostas das perguntas acima foi feita a modelagem via a técnica de storyboard. A navegação no objeto é um roteiro guiado, ou seja, um caminho pré-determinado para preservar os pré-requisitos do conteúdo. A partir do modelo a implementação foi feita em Flash e a distribuição via web.

\section{Objeto de Aprendizagem: Proporcionalidade e Semelhança}

O objeto, Proporcionalidade e Semelhança, aborda os conteúdos relacionados à razão, semelhança e proporcionalidade e prevê o conhecimento de frações, números decimais e conceitos geométricos referentes às figuras planas ou espaciais. Trata-se de um objeto que oportuniza a compreensão do que vem a ser figuras semelhantes, no sentido matemático. Muitas vezes o que é tido como semelhante, no sentido usual, não atende a alguns dos aspectos conceituais da Matemática.

O objetivo do objeto é ensinar os conceitos de razão, proporção e semelhança. Para isso será proposto ao aluno que faça ampliações ou reduções em uma foto $3 \times 4$.

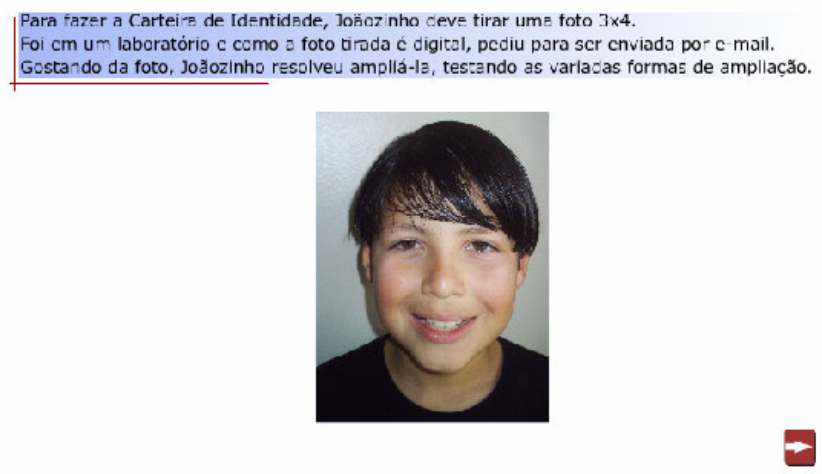

Figura 1 - Contextualização

Será preciso modificar as dimensões da foto original. Essas modificações poderão ser ou não, fiéis a foto dada e obter, assim, uma figura semelhante, no sentido matemático. Essa atividade pode servir como uma motivação ou um desafio para crianças que ainda não dominam com segurança os conceitos matemáticos nela envolvidos. O aluno é levado a alterar as dimensões da foto e verificar o que acontece segundo sua ação. 


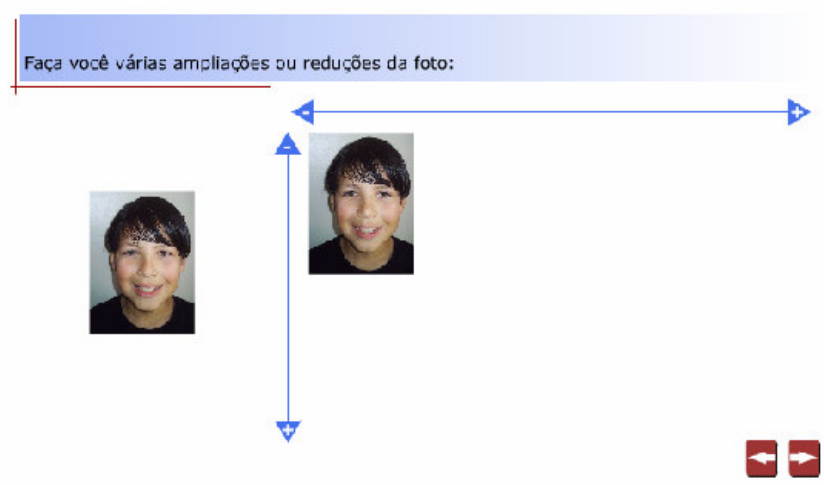

Figura 2 - Modificar as dimensões

O uso do objeto propicia que o aluno seja capaz de: aplicar conceitos geométricos; comparar figuras para identificar a semelhança; fazer ampliações e / ou reduções. O uso das animações pode proporcionar ao aluno a visualização de diferentes situações que auxiliam no aprendizado dos conceitos de semelhança, proporção e razão.

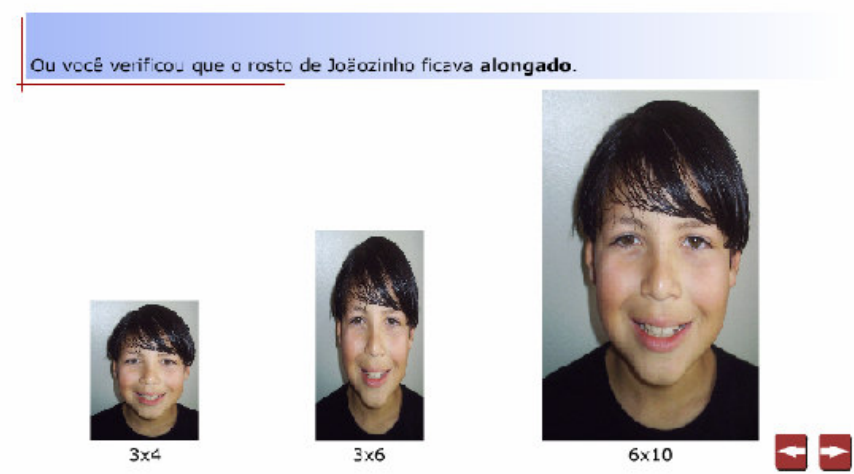

Figura 3 - Rosto modificado (alongado)

Nesse caso a ampliação não é fiel à foto original. Por meio das animações, nas atividades experimentais o aluno poderá aplicar ou rever alguns conceitos como: a descoberta da razão de semelhança utilizada em modelos; a relação entre as medidas do desenho e do real; ampliação ou redução de figuras a partir de outras. Espera-se que o aluno seja capaz de fazer conexões entre objetos semelhantes, no sentido matemático.

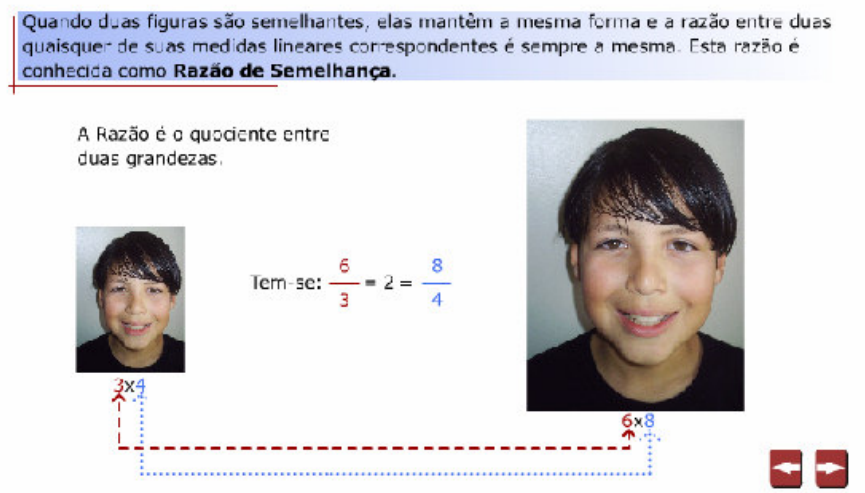

Figura 4 - Razão de Semelhança

A atividade proposta pode oportunizar o interesse do aluno em saber em que outros momentos ele se deparou com o uso das idéias envolvidas com o que ele aprendeu. $\mathrm{O}$ aluno passa a ter exemplos do uso de proporcionalidade e semelhança, em vários contextos do dia-a-dia. Aplicam-se os conceitos de semelhança e proporção quando se examinam mapas, plantas baixas de imóveis, projetos em geral, tais como móveis, 
componentes eletrônicos, mobiliário de ambientes, ampliação e redução de objetos de natureza diversa, como quadros de imagens/ fotos.

Para facilitar a aprendizagem do conteúdo do Objeto de Aprendizagem foi preciso estabelecer interatividade. A interatividade é realizada por meio de atividades digitais, que permitem construir conceitos geométricos necessários para a elaboração de modelos, tais como: a comparação entre medidas de figuras geométricas para identificar a semelhança que existe entre elas; a comparação entre elementos de uma seqüência de medidas para determinar a razão de semelhança e a proporcionalidade entre elas; a ampliação ou redução de figuras geométricas a fim de acomodá-las em um espaço prédeterminado. Por meio das interações é que os sujeitos desencadeiam trocas, estratégias de construção/autoria, compartilhamento de idéias, possibilitando gerar novas interações.

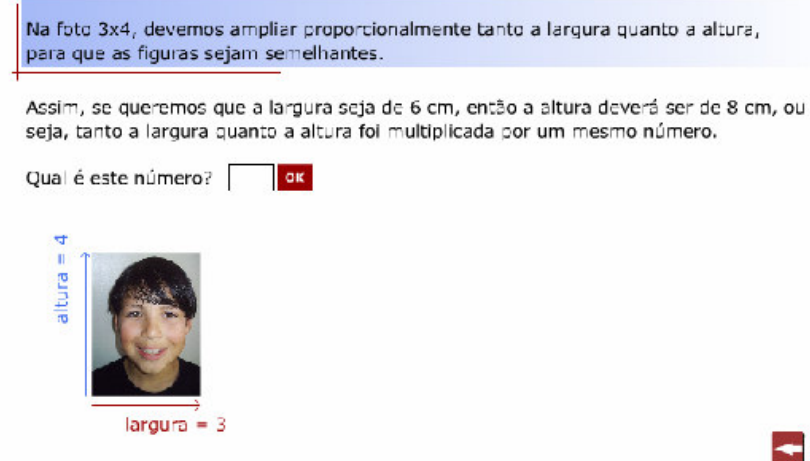

Figura 5 - Atividade

O termo interação segundo Tajra, 2002, é composto de um prefixo derivado do latim (inter) e um núcleo, também derivado do latim (actiõ - ação) que possui os respectivos significados: inter - entre, no meio de; ação: atuação, ato, feito, obra. As tecnologias digitais têm um papel fundamental ao propiciar o uso de ferramentas que apóiam e possibilitam a comunicação e a interação entre os sujeitos. As Novas Tecnologias da Informação e da Comunicação por permitirem a interação se constituem em ferramentas poderosas para a Educação, pois auxiliam na construção do conhecimento de um conteúdo, fornecendo feedback imediato e reforço dos assuntos trabalhados. Possibilitam o desenvolvimento de Objetos de Aprendizagem que podem ser utilizados em qualquer modalidade de ensino. Para garantir a eficiência de um Objeto de Aprendizagem como auxiliar didático é preciso que o professor determine quando usar esse recurso e como usar para que os objetivos do processo de ensino e aprendizagem sejam alcançados.

\section{Considerações finais}

É preciso considerar os aspectos educacionais e os objetivos que se pretende alcançar com o uso de um Objeto de Aprendizagem independente de uma definição mais técnica ou mais simples do que seja um Objeto de aprendizagem.

Com o objeto Proporcionalidade e Semelhança o aluno deverá ser capaz de aplicar conceitos geométricos, comparar figuras para identificar a semelhança, fazer ampliações/reduções, ou seja, espera-se que o aluno seja capaz de fazer conexões entre objetos semelhantes, no sentido matemático, tal como: a descoberta da razão de semelhança utilizada em modelos; a relação entre as medidas do desenho e do real; ampliação ou redução de figuras a partir de outras. $O$ uso das animações pode proporcionar ao aluno a visualização de diferentes situações que auxiliam no aprendizado dos conceitos de semelhança, proporção e razão e esses conceitos podem auxiliar a abordagem de outros conteúdos, como: 
- Interpretar e utilizar diferentes formas de representação de objetos;

- Identificar, representar e utilizar o conhecimento geométrico para aperfeiçoamento da leitura, da compreensão e da ação sobre a realidade;

- Procurar e sistematizar informações relevantes para a compreensão da situação problema;

- Entender e aplicar métodos e procedimentos próprios das ciências naturais e tecnológicas;

- Comparar e estabelecer relações entre as grandezas;

- Estimular a percepção dos alunos com relação a conceitos matemáticos envolvidos na situação-problema e levá-los a elaborar construções matemáticas abstratas para resolver problemas relativos a situações específicas, mostrando a fundamentação matemática que deve ser empregada.

As atividades devem ser desenvolvidas individualmente, os alunos terão um feedback imediato notificando o acerto ou o erro e o professor pode/deve propor discussões em grupo para fixação dos conceitos, tal como:

- Você já ouviu falar sobre o número de ouro?

- Você sabe qual é, aproximadamente, o valor da razão entre o comprimento e o diâmetro de um círculo?

- Você sabe qual a proporção que Tales usava para determinar a altura de uma pirâmide?

- Todos os retângulos são semelhantes?

- Todos os quadrados são semelhantes?

- Todos os triângulos são semelhantes?

- Todos os triângulos eqüiláteros são semelhantes?

- Todos os triângulos isósceles são semelhantes?

O professor pode levar mapas para a sala de aula e pedir para que os alunos determinem a distância, em linha reta, entre duas cidades indicadas nos mapas, sabendo que a distância no mapa é proporcional à distância correspondente na realidade.

A utilização do objeto pode ser feita também por estudantes e profissionais de engenharia, arquitetura, geografia, artistas plásticos, que trabalham com conceitos geométricos, confecção de mapas, redução/ampliação de objetos e/ou figuras.

A partir da experiência desse trabalho interdisciplinar, dos esforços da equipe, ficou constatado que o conceito de objetos de aprendizagem e seu processo de implementação podem auxiliar muito qualquer modalidade de ensino e o desenvolvimento de novos cursos e de materiais educacionais mais bem elaborados visando facilitar o processo de ensino e aprendizagem de um conteúdo.

\section{Referências Bibliográficas}

DANTE, Luiz R. Tudo é matemática. São Paulo: Ática, 2004. 6ª e 7ª́ries. DOWNES, Stephen. Learning Objects: Resources for Distance Education Worldwide. IN: Internacional Review of Research in Open and Distance Learning. Vol. 2, No. 1, 2001.

FALKEMBACH, Gilse Morgental; Cconcepção e desenvolvimento de material educativo digital - Revista Novas Tecnologias na Educação - CINTED - Centro Interdisciplinar de Novas Tecnologias na Educação - Vol. 3 No 1 (março/abril) (ISSN 1679-1916). UFRGS, 2005.

FLORES, M.L., SANTOS, L.. REIS, F. FALKEMBACH, Gilse Morgental - COASE - Criação de Objetos de Aprendizagem e suporte ao ensino - RENOTE - Revista Novas Tecnologias na Educação - CINTED - Centro Interdisciplinar de Novas Tecnologias na Educação - Vol. 4 No 1 (ISSN 1679-1916). UFRGS, 2006. 
HANDA, Jaime Kenji; SILVA, Jaime Balbino ; Objetos de Aprendizagem (Learning Objects) São paulo: boletim EAD - Unicamp, 2003

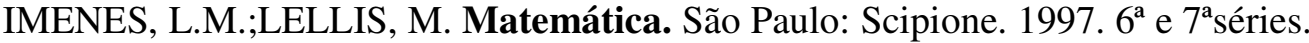

KOPER, Rob. Modeling Units of Study From a Pedagogical Perspective: the pedagogical meta-model behind EML. Open University of Netherlands, junho de 2001.

SOSTERIC, Nike; HESEMEIER, Susan. When is a Learning Object not an Object:

a first step towards a theory of learning objects. IN: Internacional Review of Research in Open and Distance Learning. Outubro de 2002.

TAJRA, Samya Feitosa. Comunidades Virtuais: Um fenômeno na Sociedade do conhecimento. São Paulo: Ed. Érica, 2002.

TAROUCP, Liane;'’TAMUSIUNAS, F. R.; FABRE, Marie. Reusabiliddae de Objetos de Educacionais, RENOTE - Revista Novas Tecnologias na Educação - CINTED Centro Interdisciplinar de Novas Tecnologias na Educação - Vol. 1 No 1 UFRGS, 2003. TINOCO, Lúcia A. A., coord. Razões e Proporções. Rio de Janeiro, UFRJ ( Instituto de Matemática), Projeto Fundão, SPEC/PADCT/CAPES, 1996.

WILEY, David A. Learning object and sequencing theory. Tese de doutorado defendida na Brigham Young University. Junho de 2000.

Especificaços da IEEE: http://ltsc.ieee.org/doc e do IMS: http://www.imsproject.org CESTA pode ser acessado via: http://www.cinted.ufrgs.br/CESTA.

RIVED pode ser acessado via: http://www.eproinfo.mec.gov.br. 